\title{
Diseño de sistemas de energía solar fotovoltaica para usuarios residenciales en Chía, Cundinamarca
}

\author{
Ana Katherine Rodríguez Manrique, ${ }^{1}$ Ángela Inés Cadena Monroy ${ }^{2}$ \\ y Andrés Julián Aristizábal Cardona* ${ }^{3 *}$
}

\author{
'Universidad de los Andes, Ingeniería Eléctrica, kath-rod@uniandes.edu.co \\ 2 Universidad de los Andes, acadena@uniandes.edu.co. \\ ${ }^{3}$ Universidad de Bogotá Jorge Tadeo Lozano, Departamento de Ingeniería, Bogotá, Colombia \\ *Autor para correspondencia: andresj.aristizabalc@utadeo.edu.co
}

\section{Resumen}

En este documento presentamos un análisis técnico y económico para el uso residencial de sistemas fotovoltaicos interconectados a la red eléctrica (BIPVS por sus siglas en inglés) para usuarios estrato 6 en el municipio de Chía, Cundinamarca.

Los estudios fueron realizados para tres tipos de vivienda de una constructora para cubrir el $100 \%, 70 \%$ y $40 \%$ de los perfiles de carga de las viviendas.

Los resultados indican que, gracias a las nuevas políticas gubernamentales (Ley 1715 de 2014) para incentivar tecnologías de generación no convencional, la recuperación de la inversión inicial del proyecto con las condiciones actuales puede llevarse a cabo en alrededor de 7 años y medio para el $100 \%$ de la demanda residencial analizada.

Palabras clave: sistemas fotovoltaicos, energía solar, generación distribuida.

Editora: Pataquiva-Mateus, A. Y.

Citation: Rodríguez, A., Cadena, A., y Aristizábal, J. (2015). Diseño de sistema de energía solar fotovoltaica para usuarios residenciales en Chía, Cundinamarca. Revista Mutis 5(1); pag 55-65.

Received: April 20, 2015; Accepted: May 20, 2015; Published on line: June 30, 2015

Copyright: (c2015 Rodríguez et al. This is an open-access article, which permits unrestricted use, distributions and reproduction in any medium, provided the original author and source are credited.

Competing Interests: The authors have no conflict of interest.

\section{Design of photovoltaic solar energy systems for residential users in Chía, Cundinamarca}

\begin{abstract}
Summary In this paper we present a technical and economic analysis for residential PV systems interconnected to the grid (BIPVS - for its acronym in English) for users layer 6 in the town of Chia, Cundinamarca.

The studies were carried out for three types of housing construction to cover $100 \%, 70 \%$ and $40 \%$ load profiles of homes.
\end{abstract}


The results indicate that thanks to new government policies (Law 1715 of 2014) to encourage nonconventional generation technologies; the recovery of the initial investment of the project with the current conditions can be carried out at about 7 and a half years for $100 \%$ of the analyzed residential demand.

Keywords: photovoltaic systems, solar energy, distributed generation.

\section{Introducción}

Con el creciente uso de la generación de electricidad distribuida a nivel mundial, se hacen necesarios estudios que contemplen la viabilidad tanto técnica como económica de la instalación y uso de fuentes de energía renovable a nivel residencial.

Con el fin de apoyar e incentivar el uso de este tipo de generación de energía, diversos países han implantado estrategias económicas que contemplan la financiación inicial de los proyectos, así como también la compra del kWh generado de forma distribuida, a precios que alcanzan a triplicar el costo del kWh generado térmica o hidráulicamente.

La energía fotovoltaica es una tecnología que ha tomado fuerza a nivel mundial en los últimos años. Los países desarrollados se están preocupando por el cuidado del medio ambiente y se han focalizado en generar energía con fuentes renovables. La energía solar fotovoltaica no emite ruido, emisiones dañinas o gases contaminantes, no necesita mantenimiento después de instalada y se puede conectar directamente a la red de forma descentralizada. Esta tecnología se ha venido difundiendo en muchos países que tienen metas de reducción de gases efecto invernadero (GEI) y se espera que los costos de inversión e instalación -su principal barrera de entrada-, desciendan rápidamente.

En este trabajo se pretende, en primer lugar, evaluar la viabilidad técnica y económica de implementar sistemas fotovoltaicos integrados en proyectos de vivienda (BIPV por sus siglas en inglés - Building Integrated Photovoltaic System); y en segundo lugar sensibilizar a los constructores y operadores de red sobre la posibilidad de adelantar proyectos demostrativos de introducción de energía fotovoltaica en el mercado colombiano.
Colombia cuenta con niveles importantes de radiación solar, el promedio multianual de la radiación solar global es de $4,5 \mathrm{kWh} / \mathrm{m}^{2}$, destacándose la península de la Guajira con un valor promedio de 6,0 kWh/ $\mathrm{m}^{2}$ y la Orinoquía con un valor un poco menor. En la sabana de Bogotá la radiación global está entre 3,57 $\mathrm{kWh} / \mathrm{m}^{2}$ y $4 \mathrm{kWh} / \mathrm{m}^{2}$, lo que significa que las horas de sol estándar son superiores a 3,57.

En el caso colombiano, además del alto costo de la inversión, habría que eliminar otra barrera, regulatoria, para posibilitar la venta de electricidad a la red por parte de los consumidores. Hay un mercado mayorista y un mercado minorista de electricidad en el país. El mercado de generación eléctrica en Colombia tiene 4 formas de transar la energía: un mercado spot, un mercado de contratos bilaterales, un mercado de confiabilidad y un mercado de reservas. La producción se realiza mediante un despacho de generación centralizado, en el que participan plantas en su mayorías hidráulicas y térmicas (gas y carbón) mayores de 10 o $20 \mathrm{MW}$, que hacen sus ofertas de disponibilidad y precio y producen según su mérito o competitividad. Las energías alternativas tienen dificultades para competir por sus mayores costos de inversión, como se dijo; y las intermitentes no podrían contar con un cargo por confiabilidad, la señal que busca garantizar la expansión en el país. El mercado minorista está muy poco desarrollado, y se anticipa que la masificación de medidores inteligentes, muy avanzada en otros países, facilite la participación de la demanda y operaciones de compraventa de energía.

En este trabajo se evalúan distintos escenarios para identificar la viabilidad y factibilidad técnica y económica de una instalación fotovoltaica que supla energía eléctrica para una urbanización en la sabana de Bogotá, más específicamente en un conjunto residencial en Chía. Para comenzar se evalúan diferentes tecnologías y proyectos existentes, así como los incentivos a nivel internacional y nacional. Los resultados indican que los aún altos costos de inversión no facilitan una penetración amplia de estos sistemas y se propone la realización de proyectos demostrativos impulsados por constructores y operadores de red como mecanismo para introducir la energía fotovoltaica al mercado colombiano y mostrar su factibilidad para alimentar consumos residenciales e intercambiar energía con la red de distribución eléctrica. 
El artículo se encuentra organizado de la siguiente forma: en el capítulo 2 se realiza la selección del punto de ubicación de los sistemas fotovoltaicos, así como también el diseño técnico de los mismos. En el capítulo 3 se evalúa la viabilidad técnica y económica del proyecto y en el capítulo 4 se presentan las conclusiones del estudio.

\section{Selección de la ubicación y dimension- amiento del proyecto}

Con el fin de cumplir con los objetivos propuestos en el proyecto, fue necesario seleccionar una ubicación específica para el desarrollo del mismo. El lugar escogido para tal fin fue el municipio de Chía, aprovechando su cercanía a uno de los proyectos piloto de Colombia para así facilitar las investigaciones relacionadas con las mediciones de radiación solar en la zona. La urbanización en la que se realizaron los estudios está ubicada vía a Cajicá, km 2 al norte de Centro Chía.

Para el desarrollo de este proyecto fue necesario identificar previamente las etapas en las que este estaría dividido. Las primeras etapas identificadas a estudiar fueron las siguientes:

- Disponibilidad del recurso energético en la sabana de Bogotá.

- Dimensionamiento técnico e instalación del sistema fotovoltaico interconectado para varios casos (base y escenarios).

- Comportamiento de la red eléctrica frente a la instalación de un sistema fotovoltaico interconectado.

\section{Disponibilidad del recurso energético}

En esta primera etapa se investigó el recurso energético solar disponible en la sabana de Bogotá. Para esto fue necesario acudir al Atlas de Radiación Solar de la UPME en donde se evidencia que el promedio multianual de la radiación solar global en la sabana de Bogotá es de $4 \mathrm{kWh} / \mathrm{m}^{2}$ (ver figura 1). Adicionalmente a este dato, el Grupo de Materiales Semiconductores y Energía Solar de la Universidad Nacional de Colombia ha realizado investigaciones para un proyecto piloto ubicado en la Universidad Nacional de Colombia sede Bogotá, en el que encontraron que la radiación solar en la sabana de Bogotá es de $3,57 \mathrm{kWh} / \mathrm{m}^{2}$. Lo que significa que las horas de sol estándar en Chía son 3,57 HSS.

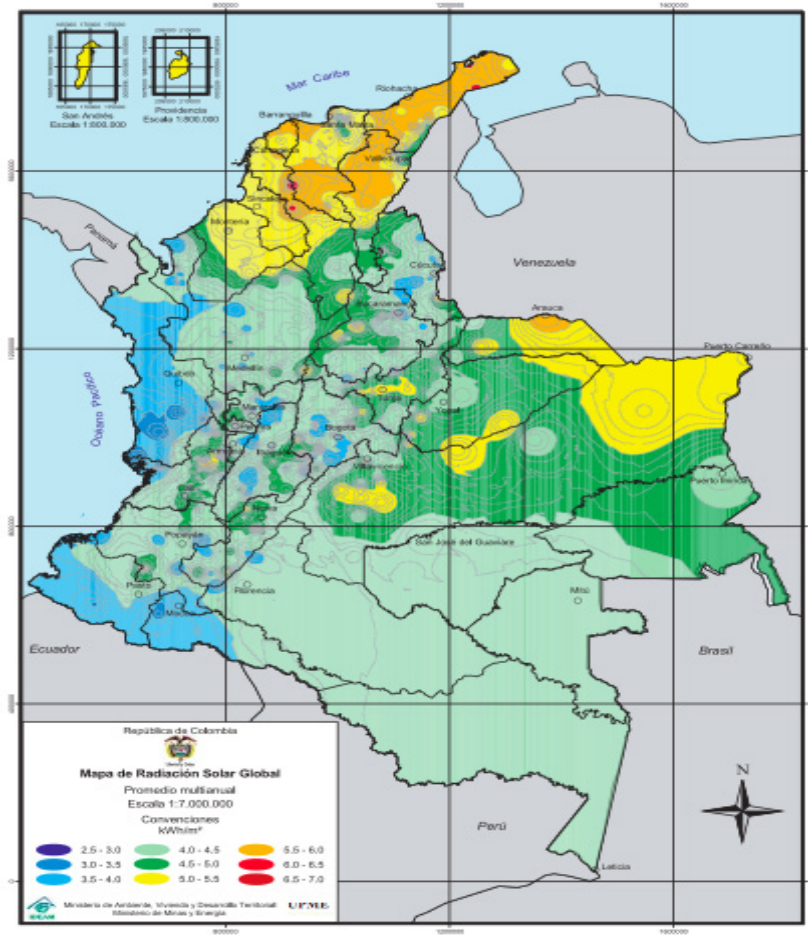

Figura 1. Mapa de radiación solar global

Para estudiar la disponibilidad del recurso energético en la sabana de Bogotá y el dimensionamiento técnico del sistema, se utilizaron los datos analizados por el Grupo de Materiales Semiconductores y Energía Solar de la Universidad Nacional de Colombia. Posteriormente fue estudiada la viabilidad de instalación en la urbanización gracias a la información entregada por parte de la constructora.

\section{Dimensionamiento técnico y diseño del sistema fotovoltaico para un caso base}

Esta es una de las etapas más importantes del proyecto, pues en esta se definen la carga del sistema y los elementos a instalar. También se analiza el impacto que tiene la instalación fotovoltaica sobre la construcción.

\section{Dimensionamiento técnico}

Existen dos manera de dimensionar el sistema fotovoltaico: la primera, partiendo de la disponibilidad económica para determinar la capacidad de potencia de la planta y la otra partiendo de la demanda de energía para conocer el costo total de la instalación. Para llevar a cabo el dimensionamiento técnico de este proyecto, se partió de la demanda energética promedio de una casa de estrato 6 ubicada en Chía. 
Tabla 1. Consumo mensual promedio y valor facturado por unidad de consumo de los últimos tres años para el sector residencial de estrato 6 en Chía

\begin{tabular}{|c|c|c|c|c|}
\hline Año & Estrato & № de usuarios & $\begin{array}{c}\text { Consumo } \\
\text { promedio anual } \\
\text { (kWh) }\end{array}$ & $\begin{array}{c}\text { Valor facturado } \\
\text { por unidad de } \\
\text { consumo (\$) }\end{array}$ \\
\hline 2012 & \multirow{3}{*}{6} & 936 & 5148 & 338,64 \\
\hline 2013 & & 987 & 5547 & 346,45 \\
\hline 2014 & & 1036 & 5636 & 361,27 \\
\hline
\end{tabular}

La tabla 1 muestra los consumos promedio mensuales de los últimos tres años para una casa de estrato 6 en Chía, los cuales promedian un valor aproximadamente de 453,64 kWh-mes. Con este dato se puede encontrar la potencia que debe generar el generador fotovoltaico (módulos fotovoltaicos) para suplir la carga de una casa estrato 6 en Chía.

$$
P_{G F V}=\frac{E}{H S S^{*} N * P R}
$$

Ecuación 1. Potencia a la salida del generador fotovoltaico

Con la ecuación 1 se puede encontrar la potencia que deberá suplir el generador fotovoltaico a partir del consumo promedio mensual investigado anteriormente, donde:

E: energía consumida mensualmente (kWh-mes).

HSS: horas de sol estándar de la zona (para este caso 3,57h).

$\mathrm{N}$ : número de días en el que consume la energía $\mathrm{E}$ (30 días).

PR: factor de rendimiento del sistema $(0,7$ a 0,8$)$.

\section{Inversor}

Según la ecuación 1 , la potencia que debe producir el generador fotovoltaico es de $5,3 \mathrm{~kW}$, es decir, esta es la potencia que debe haber a la salida del inversor. Conociendo esta potencia, se puede iniciar la búsqueda del inversor en el mercado. Una vez se ha seleccionado un inversor, los parámetros de más importancia a tener en cuenta son la potencia de entrada del mismo y su punto máximo de potencia (MPP).

Para este proyecto se realizó el dimensionamiento con un inversor Sunny Boy 5000US 208Vac, el cual necesita a la entrada una potencia máxima de $6,2 \mathrm{~kW}$ y tiene un punto máximo de potencia de 250 a $480 \mathrm{~V}$ (tabla 2).

Tabla 2. Parámetros eléctricos Iversor Sunny Boy 5000US a 208V

\begin{tabular}{|c|c|}
\hline \multicolumn{2}{|l|}{$\begin{array}{l}\text { Sunny Boy 5000US 208V } \\
\text { Información técnica }\end{array}$} \\
\hline Máxima potencia PV recomendada & $6250 \mathrm{~W}$ \\
\hline Máximo voltaje DC & $600 \mathrm{~V}$ \\
\hline Punto de máxima potencia & $250-480 \mathrm{~V}$ \\
\hline Corriente máxima de entrada DC & $21 \mathrm{~A}$ \\
\hline Potencia nominal AC & $5000 \mathrm{~W}$ \\
\hline Máxima potencia de salida AC & $5000 \mathrm{~W}$ \\
\hline Máxima corriente de salida & $24 \mathrm{~A}$ \\
\hline Rango voltaje nominal AC & $183-229 \mathrm{~V}$ \\
\hline Frecuencia AC & $60 \mathrm{~Hz}$ \\
\hline
\end{tabular}




\section{Módulos fotovoltaicos}

La potencia a la entrada del inversor será la potencia necesaria a la salida de los módulos fotovoltaicos, es decir, deben instalarse un número de paneles tal que generen $6,2 \mathrm{~kW}$.

El módulo fotovoltaico seleccionado para este proyecto fue el FE180M, el cual genera una potencia de 180 W, tiene un voltaje máximo de $35,25 \mathrm{~V}$ y una corriente máxima de 5,11 A (tabla 3).

Tabla 3. Información técnica panel solar monocristalino FE180M-180W

\begin{tabular}{|l|r|}
\hline \multicolumn{2}{|c|}{ Panel solar monocristalino FE180M-180W } \\
Información técnica \\
\hline Máxima potencia & $180 \mathrm{~W}$ \\
\hline Tolerancia & $+/-3 \%$ \\
\hline Corriente de máxima potencia (A) & $5,11 \mathrm{~A}$ \\
\hline Tensión de máxima potencia (V) & $35,25 \mathrm{~V}$ \\
\hline
\end{tabular}

\section{Generador fotovoltaico}

El número de paneles de $180 \mathrm{~W}$ necesarios para alcanzar una potencia de $7,5 \mathrm{~kW}$ es de 35 paneles (se usarán 36 por conexiones eléctricas). Una vez se halla el número de paneles a instalar se prosigue a configurarlos en serie (sumando voltaje) o en paralelo (sumando corriente). Estos 36 paneles se configuraron de tal manera que el voltaje de salida (Vmp) estuviera dentro del rango del punto máximo de potencia del inversor, encontrandose que se deben instalar 4 configuraciones en paralelo de 9 paneles en serie para tener un voltaje máximo pico a la salida de la configuración fotovoltaica de 317,25 V.

Con esta configuración los 36 paneles generan una potencia de 6,4 kW, potencia suficiente para entregar al inversor.

\section{Tablero general:}

El tablero general representa el punto de acople común (PCC) donde se realiza la conexión en paralelo entre el sistema fotovoltaico a la red, las protecciones de corriente y los medidores de energía.
Según la norma NTC 2050, la capacidad de los conductores y la corriente nominal o ajuste de disparo de los dispositivos de protección contra sobrecorriente en el punto en que estará ubicada la protección, no debe ser menor al $125 \%$ de la corriente nominal a la salida del inversor. Si la máxima corriente de salida del inversor Sunny Boy 6000US 208Vac es 29 A, es indispensable utilizar una protección de 36 A mínimo.

\section{Sistema de puesta a tierra}

Según la norma NTC 2050, en todas las fuentes fotovoltaicas debe haber un conductor de un sistema bifilar de más de $50 \mathrm{~V}$ nominales y el conductor neutro de un sistema trifilar que estén sólidamente puestos a tierra. Se debe colocar un punto de conexión de puesta a tierra lo más cercano posible de la fuente fotovoltaica, así el sistema quedará mejor protegido contra las posibles subidas de tensión producidas por los rayos. Las estructuras metálicas de los equipos que componen el sistema también deberán estar aterrizadas a tierra.

\section{Diseño del sistema fotovoltaico}

Cuando se habla en el proyecto del diseño del sistema fotovoltaico, se pretende estudiar el impacto que podría tener la instalación de los paneles solares y demás elementos en las casas de la constructora analizada. Para esto se debe tener en cuenta que el sistema que trata el proyecto es un sistema fotovoltaico integrado a una construcción (BIPVS), por lo que es ideal instalar los paneles en un techo plano.

Se escogieron tres modelos de casas en esta urbanización, con el fin de hacer simulaciones de la instalación de los paneles fotovoltaicos.

- C- Cedro: tiene un área de 57,95 $\mathrm{m}^{2}$ de techo plano.

- Sauce C: tiene un área de $45,39 \mathrm{~m}^{2}$ de techo plano.

- Alcaparro: tiene un área de $28,5 \mathrm{~m}^{2}$ de techo plano.

El área cuadrada de los techos planos es el criterio del proyecto para instalar una capacidad igual o menor que la demanda total de una casa, pues si el área en las casas no es lo suficientemente grande para instalar la cantidad de paneles necesarios para cubrir toda la demanda de la residencia, se tiene que contemplar la posibilidad de instalar un generador de menor capacidad. 
En cuanto a la inclinación de los paneles fotovoltaicos, esta se definió de acuerdo a estudios realizados en los que se encontró que la inclinación óptima de los paneles fotovoltaicos en zonas ubicadas en el hemisferio norte y cercanas a la línea del ecuador es la misma que la latitud de la zona. De lo anterior, se concluye según la latitud geográfica de Chía $\left(4^{\circ} 51^{\prime} \mathrm{N}\right)$, que la inclinación óptima de los paneles solares es de $4^{\circ}$ orientados hacia el sur.

Para observar el diseño de los paneles fotovoltaicos instalados en una casa, se utilizó el programa SketchUp, el cual permite ubicar la construcción diseñada en Google Earth y hacer simulaciones de sombras para la estructura según su localización.

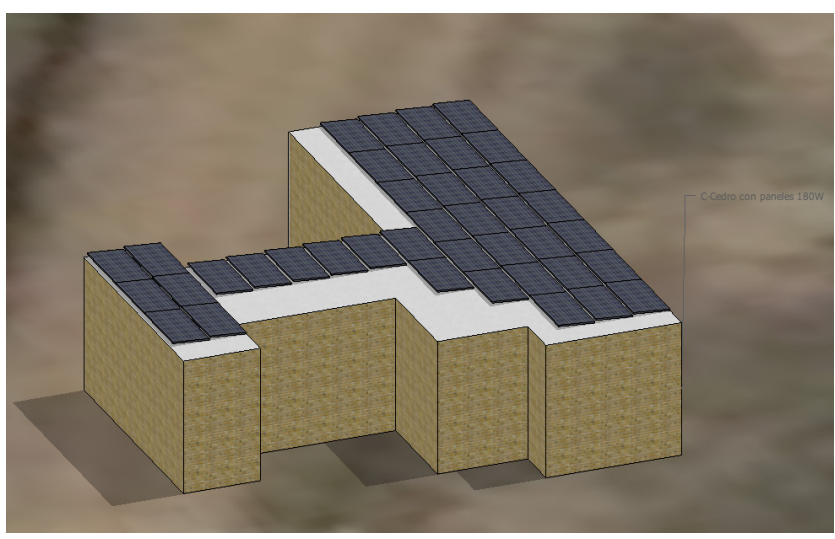

Figura 3. Diseño de la instalación con paneles fotovoltaicos en el techo plano de la casa C-Cedro

\section{Dimensionamiento técnico y diseño de sistemas fotovoltaicos para otros casos}

Se realizaron dos dimensionamientos técnicos más, teniendo en cuenta el caso base. En la sección del diseño del sistema fotovoltaico para el caso base, se estableció como criterio de la capacidad a instalar en una casa, el área disponible para la instalación de los paneles fotovoltaicos; por lo cual se llevaron a cabo los dimensionamientos para las casas Sauce C y Alcaparro que cuentan con $45,39 \mathrm{~m}^{2}$ y $28,5 \mathrm{~m}^{2}$ respectivamente.
Los datos mostrados en la tabla 4 se hallaron teniendo en cuenta las condiciones de dimensionamiento para cada uno de los equipos tal como se explicó en el caso base. Esta tabla muestra el número de paneles solares necesarios para suplir el $100 \%(453,64 \mathrm{kWh})$, el $70 \%(317,548 \mathrm{kWh})$ y el $40 \%(181,456 \mathrm{kWh})$ de la carga total promedio de una casa de estrato 6 en Chía, en donde las áreas que ocupan los paneles solares son de 51,42 y $23 \mathrm{~m}^{2}$ respectivamente.

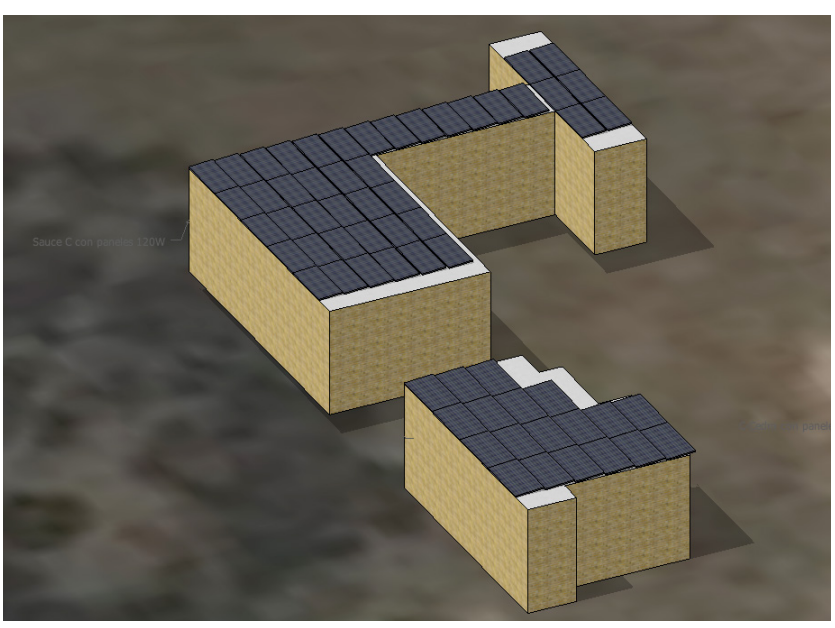

Figura 4. Diseño de las instalaciones de los paneles fotovoltaicos.

Arriba: techo plano de casa Sauce C. Abajo: techo plano de casa Alcaparro

\section{Comportamiento de la red eléctrica frente a la instalación de un sistema fo- tovoltaico}

En el caso del sistema de mayor capacidad $(6,4 \mathrm{~kW})$, el cual proporciona el $100 \%$ del consumo promedio mensual para un usuario residencial de estrato 6 en Chía, suponiendo que se instalarían 30 sistemas fotovoltaicos con esta capacidad, se le estaría entregando a la red una potencia de $192 \mathrm{~kW}$. Esta potencia es pequeña dentro del marco de referencia de la red de media tensión (11,4 kV). Por lo tanto, al instalar los 30

Tabla 4. Dimensionamientos para los 3 modelos de casas a estudiar

\begin{tabular}{|c|c|c|c|c|c|r|}
\hline Casa & Consumo (Wh) & Pin-Inversor (W) & Tipo de Panel & Num. Paneles & Area total (m2) & Precio Aproximado \\
\hline C-Cedro & 453640 & 5000 & FE180M-180W & 36 & 51,065 & $\$ 22,848,720$ \\
\hline Sauce C & 317548 & 3700 & FE180M-180W & 20 & 42,014 & $\$ 14,680,400$ \\
\hline Alcaparro & 181456 & 2500 & FE180M-180W & 12 & 22,979 & $\$ 9,096,240$ \\
\hline
\end{tabular}


sistemas, los niveles de cargabilidad y de voltaje no se modifican de manera significativa y continúan cumpliendo el lineamiento de código de redes establecido en la resolución de la CREG 025 de 1995.

\section{Viabilidad económica y regulatoria del sistema fotovoltaico}

Después de realizar el estudio de disponibilidad de recurso y estudiar el dimensionamiento técnico para tres de las casas de la constructora, se procede a realizar las siguientes dos etapas.

- Estudio de viabilidad económica del sistema.

- Estudio de la viabilidad regulatoria para la instalación.

\section{Estudio de viabilidad económica del siste- ma}

Asumiendo que el costo de un vatio fotovoltaico puesto en Bogotá es de 1 USD/W con TRM de \$2489 (22 de agosto de 2015), con una tasa de retorno del 10 $\%$ y que la vida útil de los paneles solares a implementar es de 25 años, se puede encontrar el valor del kWh fotovoltaico mensual, el cual es de \$759 COP/ kWh-mes. Si se compara este valor con el valor facturado por unidad de consumo de los últimos tres años para las casas de estrato 6 en Chía (\$348/kWh-mes), se puede observar que el costo de la energía fotovoltaica es 2,2 veces mayor al costo de la energía entregada por la red.

Los flujos de caja, entonces, dependerían de las FIT o GT entregadas al generador, las ganancias por costos evitados, los gastos de operación y mantenimiento, etc.

Todos esos factores pueden trasladarse a un flujo de caja $\mathrm{Ct}^{*}$ por medio de la ecuación 2 sumando algebraicamente todos los costos $\mathrm{Ci}$ y todos los beneficios Pi de un año genérico.

$$
\begin{aligned}
& C t^{*}=/ P_{i}-/ C_{i, t} \\
& C t^{*}=F \cdot E_{\mathrm{t}}+c_{\mathrm{kwh}, t} \cdot E_{\mathrm{t}}-u \cdot C_{\mathrm{o}}
\end{aligned}
$$

Ecuación 2. Flujo de caja total.
Donde:

\section{F: FIT o GT}

CkWh,t: precio de electricidad en un n-ésimo año.

u: coeficiente utilizado para evaluar los costos de operación y mantenimiento, usualmente iguales a 0,01 para sistemas fotovoltaicos.

co: costo de la inversión inicial.

Con el fin de hacer un análisis realista, los flujos de caja se anualizan utilizando la ecuación 3.

$$
C t=\frac{C t^{*}}{(1+i)^{t}}
$$

Ecuación 3. Flujo de caja anualizado

Con esta ecuación ya se puede encontrar el Valor Presente Neto que está dado por:

$$
V N P=/_{\mathrm{t}=1}^{\mathrm{N}} C t-C_{\mathrm{o}}
$$

Ecuación 4. VPN del flujo de caja

Con las ecuaciones presentadas anteriormente se mostrarán los cálculos de VPN para el caso en que se quiere alimentar la carga total de la casa y en el caso en el que se quiere suministrar solamente el $40 \%$ de la residencia.

\section{Caso carga completa}

Aplicando la ecuación 2 y asumiendo la variable $\mathrm{F}=0$, el precio de la energía es de $\$ 360 / \mathrm{kWh}$-mes, el consumo mensual es de 453,64 kWh-mes y la inversión inicial de $\$ 22$ '848.720. El flujo de caja Ct* tiene un valor de $\$ 352.934$.

Teniendo en cuenta los incentivos aplicados para generación de electricidad no convencional de la Ley 
1715 de 2014 y que se presentan en la tabla 5, la inversión inicial de $\$ 22^{\prime} 848.720$ tendría los siguientes descuentos:

- Exoneración del pago del IVA (16 \%): \$3’655.795.

- Exoneración del pago de derechos arancelarios: $\$ 44^{\prime} 568.744$.

De esta manera, la inversión inicial actualizada será de: \$14'623.181.

La inversión para instalar un sistema que supla la energía total de una casa se recupera en su totalidad durante los primeros siete años y medio de operación del sistema.

\section{Caso $\mathbf{4 0} \%$ de la carga}

Ahora, asumiendo que se quiere instalar el sistema de 181,45 kWh-mes, el precio de la energía es de $\$ 360 /$ kWh-mes y la inversión inicial es de $\$ 9^{\prime} 096.240$.

Aplicando los incentivos, tenemos:

- Exoneración del pago del IVA (16 \%): \$1’444.198.

- Exoneración del pago de derechos arancelarios: $\$ 1 ' 819.248$.

De esta manera, la inversión inicial actualizada será de: $\$ 5$ '832.794.

Observando los resultados para un sistema que quiere suplir el $40 \%$ de la energía que demanda la casa, se puede concluir que la inversión se recupera en el lapso de los primeros siete años de operación del sistema.

\section{Estudio de la viabilidad regulatoria para la instalación}

En el estudio de viabilidad económica mostrado anteriormente, se puede observar que la inversión inicial de la instalación del sistema fotovoltaico es muy alta en comparación con la suma de costos evitados anuales durante la vida útil de mismo, por esta razón es de gran importancia ofrecer incentivos al generador de energía, así se suman los beneficios de este a los costos evitados y aumentaría el Valor Presente Neto. Este generador se puede clasificar como generador distri- buido, por lo cual se beneficia de estudios realizados en Colombia para introducir la generación distribuida (GD) en el mercado energético colombiano.

Incentivos para el generador se están aplicando actualmente en países de la Unión Europea, entre los que están los Feed-inTariff (FiT) y los Green Tags(GT). Sin embargo, se considera que los FiT no son viables en Colombia.

Algunos de los incentivos para el país para implementar la energía fotovoltaica como generador distribuido son: el ahorro de costos de transporte (transmisión y distribución), la reducción en los niveles de pérdidas y la confiabilidad del sistema.

La Universidad de los Andes, la Universidad Nacional, Isagen y Colciencias trabajaron en el proyecto de investigación: "Regulación para incentivar las energías alternas y la generación distribuida en Colombia", en el cual se concluye que la regulación del mercado energético actual no limita la implementación de generadores a partir de energías alternativas. Esta regulación tampoco establece remuneración para el generador por la instalación de estas energías, considerando la participación de generación distribuida en las redes. Igualmente, es indispensable contar con esquemas de financiación, de tal manera que los inversionistas sean conscientes de los beneficios generados para el país relacionados con la reducción de emisiones y el desarrollo tecnológico del mismo.

La CREG, por su parte, contrató un estudio realizado por el consultor Alberto Rodríguez Hernández en noviembre del 2009 llamado: "La generación distribuida y su posible integración al sistema interconectado nacional", en el cual se exponen algunas recomendaciones y conclusiones dirigidas a la CREG acerca de las Redes Inteligentes y la Generación Distribuida. Dentro del estudio se incluyeron gráficas que muestran la participación de la energía solar en el 2030 para abastecer la demanda energética mundial y la disponibilidad de este recurso para el mismo año. El recurso solar disponible es de 580 TW, esta es una cantidad mucho mayor al recurso de agua disponible, el cual es de 2 TW.

Las recomendaciones de este estudio fueron:

- Es conveniente incluir el concepto de generación distribuida dentro de la normatividad. 
- Al tiempo se recomienda revisar el actual concepto de plantas menores.

- Se recomienda revisar las definiciones de productor marginal, productor independiente y productor para uso propio, promoviendo ajustes legales o regulatorios según corresponda.

- Se recomienda crear la actividad de generación distribuida.

- Se recomienda permitir la venta de excedentes de los autogeneradores.

- Se recomienda introducir la medición dual y las tarifas diferenciales para la energía en horas punta y horas valle.

- Desarrollar planes piloto para nuevos complejos habitacionales, nuevos edificios y nuevos centros comerciales, que se basen en los conceptos de generación distribuida, redes inteligentes y arquitectura sostenible.

Además de las recomendaciones, en este estudio Alberto Rodríguez Hernández insiste en que se requieren incentivos para desarrollar la GD, especialmente con fuentes nuevas y renovables.

El Congreso de la República expidió la Ley 697 de octubre 3 de 2001, por medio de la cual se fomenta el uso racional y eficiente de la energía, se promueve la utilización de energías alternativas y se dictan otras disposiciones.

En el año 2014, se promulga la Ley 1715 del 13 de mayo del mismo año; mediante la cual se regula la integración de las energías renovables no convencionales al Sistema Energético Nacional (SIN).

En la tabla 5 se presenta una comparación de ambas leyes en materia de incentivos.

Los incentivos que promulga la Ley 1715 de 2014 representan un avance significativo que permitirá redundar en la promoción de nuevas tecnologías energéticas no convencionales interconectadas con el SIN. También serían interesantes nuevos modelos de apoyo para este tipo de proyectos relacionados con la compra por parte de la empresa prestadora del servicio eléctrico del $\mathrm{kW}$, generado con estas nuevas fuentes, a precios que incluso duplican el costos del kWh generado convencionalmente (hidráulico o térmico) y la financiación de hasta el 70 \% de la inversión inicial del proyecto; tal como ocurre en la actualidad en países como Alemania, Japón y Estados Unidos.

\section{Conclusiones}

La viabilidad técnica depende directamente del área disponible para la instalación del sistema. Debido a la relación proporcional entre el área disponible para instalar el sistema fotovoltaico y la potencia generada por el sistema, se concretó como criterio para determinar la capacidad del generador fotovoltaico el área de techo plano en una casa.

Las Universidades de los Andes y Nacional (Minas), Isagen, Colciencias, y la CREG examinaron posibles medidas regulatorias que se podrían imponer en el país mediante estudios y análisis del mercado energético actual. En estos estudios se recomienda introducir el concepto de Generación Distribuida a la normatividad colombiana y autorizar la compraventa de energía para estos medios de generación o medición neta.

La regulación actual no limita la implementación de energías renovables pero tampoco muestra incentivos para que estas se efectúen. La UPME por su parte está desarrollando la formulación de un plan de desarrollo energético para las fuentes no convencionales de energía en Colombia (PDFNCE).

Los dimensionamientos realizados y analizados a la luz de la Ley 1715 de 2014, permiten recuperar la inversión inicial en períodos de hasta 7 años y medio de operación de los sistemas solares fotovoltaicos, convirtiendo esta tecnología en una opción viable y óptima para ser usada en generación distribuida a nivel urbano. 
Tabla 5. Comparación de los incentivos otorgados según la Ley 697 de 2001 y la Ley 1715 de 2014

\begin{tabular}{|c|c|c|}
\hline Aspecto & Ley 697 de 2001 & Ley 1715 de 2014 \\
\hline \multirow[t]{2}{*}{ Incentivos } & $\begin{array}{l}\text { Para la investigación: el gobierno nacio- } \\
\text { nal propenderá por la creación de pro- } \\
\text { gramas de investigación en el uso racio- } \\
\text { nal y eficiente de la energía a través de } \\
\text { Colciencias. }\end{array}$ & $\begin{array}{l}\text { Incentivos a la generación de energías no } \\
\text { convencionales: fomento a la investiga- } \\
\text { ción, desarrollo e inversión en el ámbito } \\
\text { de la producción y utilización de energía } \\
\text { a partir de FNCE, la gestión eficiente de la } \\
\text { energía. Los obligados a declarar renta que } \\
\text { realicen directamente inversiones en este } \\
\text { sentido, tendrán derecho a reducir anual- } \\
\text { mente de su renta por los } 5 \text { años siguien- } \\
\text { tes al año gravable en que hayan realizado } \\
\text { la inversión, el } 50 \% \text { del valor total de la } \\
\text { inversión realizada. }\end{array}$ \\
\hline & $\begin{array}{l}\text { Para la educación: el Icetex beneficia- } \\
\text { rá con el otorgamiento de préstamos } \\
\text { a los estudiantes que quieran estudiar } \\
\text { carreras o especializaciones orientados } \\
\text { en forma específica a aplicación en el } \\
\text { campo URE. }\end{array}$ & \\
\hline \multirow{3}{*}{ Incentivos } & $\begin{array}{l}\text { Incentivo tributario IVA: los equipos, } \\
\text { elementos, maquinaria y servicios na- } \\
\text { cionales o importados que se desti- } \\
\text { nen a la preinversión o inversión para } \\
\text { la producción y utilización de energía a } \\
\text { partir de las fuentes no convencionales, } \\
\text { así como para la medición y evaluación } \\
\text { de los potenciales recursos estarán ex- } \\
\text { cluidos de IVA. }\end{array}$ & \\
\hline & $\begin{array}{l}\text { Reconocimiento público: el gobierno } \\
\text { nacional creará distinciones para per- } \\
\text { sonas naturales o jurídicas, que se des- } \\
\text { taquen en el ámbito nacional en apli- } \\
\text { cación del URE, las cuales se otorgarán } \\
\text { anualmente. El Ministerio de Minas y } \\
\text { Energía dará amplio despliegue a los } \\
\text { galardonados en los medios de comu- } \\
\text { nicación más importantes del país. }\end{array}$ & $\begin{array}{l}\text { Incentivo arancelario: las personas natura- } \\
\text { les o jurídicas que a partir de la vigencia } \\
\text { de esta ley sean titulares de nuevas inver- } \\
\text { siones en nuevos proyectos de FNCE, goza- } \\
\text { rán de exención del pago de los derechos } \\
\text { arancelarios de importación de maquina- } \\
\text { ria, equipos materiales e insumos que no } \\
\text { sean producidos por la industria nacional y } \\
\text { su único medio de adquisición esté sujeto } \\
\text { a la importación de los mismos. }\end{array}$ \\
\hline & $\begin{array}{l}\text { Generales: el gobierno nacional esta- } \\
\text { blecerá los incentivos e impondrá las } \\
\text { sanciones de acuerdo con el programa } \\
\text { de uso racional y eficiente de la energía } \\
\text { y demás formas de energía no conven- } \\
\text { cionales, de acuerdo a las normas lega- } \\
\text { les vigentes. }\end{array}$ & $\begin{array}{l}\text { Incentivo contable: depreciación acelera- } \\
\text { da de activos. La actividad de generación } \\
\text { de electricidad a partir de FNCE, gozará del } \\
\text { régimen de depreciación acelerada. }\end{array}$ \\
\hline
\end{tabular}




\section{Referencias}

Aristizábal, A., Botero, M., \& Gordillo, G. (2010). Evaluación del desempeño energético de un sistema BIPVS de 3,4kW y Dimensionamiento de un sistema BIPVS para un usuario doméstico típico en Bogotá, Colombia. Segunda Conferencia Iberoamericana de Ingeniería e Innovación Tecnológica, Orlando, USA.

Ayompe, L. M., Duffy, A., McCormack, S. J., \& ConIon, M. (2010). Projected costs of a grid-connected domestic PV system under different scenarios in Ireland, using measured data from a trial instaIlation. Energy Policy, 38(7): 3731-3743.

Bayod-Rújula, A. A., Ortego-Bielsa, A., \& Martínez-Gracia, A. (2011). Photovoltaics on flat roofs: energy considerations. Energy, 36(4): 1996-2010.

Cadena, A. (2010). Viabilidad de Energías Renovables en la Generación Eléctrica en Colombia. Universidad de los Andes y Ministerio de Minas y Energía, Bogotá.

Campoccia, A., Dusonchet, L., Telaretti, E., \& Zizzo, G. (2009). Comparative analysis of different supporting measures for the production of electrical energy by solar PV and Wind systems: Four representative European cases. Solar Energy, 83(3): 287-297.

Cheng, C. L., Jiménez, C. S. S., \& Lee, M. C. (2009). Research of BIPV optimal tilted angle, use of latitude concept for south orientated plans. Renewable Energy, 34(6): 1644-1650.
Du H., Li N., Brown M. A., Peng Y., \& Shuai Y. (2014). A bibliographic analysis of recent solar energy literatures: the expansion and evolution of a research field. Renewable Energy, 66: 696-706.

Instituto Colombiano de Normas Técnicas y Certificación. (1998). Código Eléctrico Colombiano NTC 2050.

Quiceno, G. (2014). Cuadro comparativo entre la Ley 697 de 2001 y la Ley 1715 de 2014", Universidad de Bogotá Jorge Tadeo Lozano.

Sistema Único de Información de Servicios Públicos Retrieved 06/05, (2015), from http://www.sui.gov. co/SUIAuth/logon.jsp

Universidad de los Andes y Grupo Endesa, (2010). Cambio climático, Diagnostico, perspectivas y lineamientos para definir estrategias posibles ante el cambio climático, Bogotá.

UPME Retrieved 15/09, (2010), from http://institucional.ideam.gov.co/jsp/info/institucional/media/ descargas/atlas_radiacions/1-Preliminares.pdf

UPME Retrieved 15/09, (2010), from http://www. upme.gov.co/Docs/Atlas_Radiacion_Solar/1-Atlas_Radiacion_Solar.pdf 